\title{
Introduction to Lumbosacral and Sacropelvic Fixation Strategies
}

\author{
Patrick C. Hsieh, M.D. ${ }^{1}$ and Praveen V. Mummaneni, M.D. ${ }^{2}$
}

${ }^{1}$ Department of Neurological Surgery and USC Spine Center, University of Southern California Keck School of Medicine, Los Angeles, California; and 'Department of Neurosurgery and UCSF Spine Center, University of California, San Francisco, California

We are pleased to present this Neurosurgical Focus video supplement on lumbosacral and sacropelvic fixation strategies. Despite advancement in surgical techniques and technologies in spine, achieving consistent solid fusion across the lumbosacral junction remains a major challenge. The anatomy of the lumbosacral junction allows for a higher range of motion compared to other areas of the thoracolumbar spine. The L5-S1 interspace is exposed to significant shear forces. As a result, complications such as pseudoarthrosis, screw pull-out, implant fracture, or sacral fractures can occur. Complications are particularly seen in long fusion constructs ending across the lumbosacral junction. To reduce these complications, various lumbosacral and sacropelvic fixation techniques have been developed and utilized.

The current supplement is intended to provide instructional videos that illustrate several current techniques for lumbosacral and sacropelvic fixation. The collection includes techniques for anterior L5-S1 interbody fusion, minimally invasive L5-S1 interbody fusions, lumbosacral pedicle screw placement, sacroiliac fusion, and sacro-alar-iliac screw placement. The authors of the videos in the supplement have provided detailed narration and video illustration to describe the nuances of the various open and minimally invasive techniques for lumbosacral and sacral-pelvic fixation. We are pleased to have such a collection of quality video illustration from experts in the field. It's been our privilege to serve as guest editors for this supplement and we believe that you will enjoy the contents of this supplement. 